\title{
Quantum modeling of two-level photovoltaic systems
}

\author{
Tahereh Nemati Aram ${ }^{1,2, a}$, Asghar Asgari ${ }^{2,3}$, Matthias Ernzerhof ${ }^{4}$, Pascal Quémerais ${ }^{1}$, and Didier Mayou ${ }^{1}$ \\ 1 Institut NÉEL, CNRS and Université Grenoble Alpes, 38042 Grenoble, France \\ 2 Research Institute for Applied Physics and Astronomy, University of Tabriz, 51666-14766 Tabriz, Iran \\ 3 School of Electrical, Electronic and Computer Engineering, The University of Western Australia, WA 6009 Crawley, Australia \\ 4 Département de Chimie, Université de Montréal, C.P. 6128 Succursale A, Montréal, Québec H3C 3J7, Canada
}

Received: 21 November 2016 / Received in final form: 23 February 2017 / Accepted: 3 May 2017

(c) T. Nemati Aram et al., published by EDP Sciences, 2017

\begin{abstract}
We present a quantum formalism that provides a quantitative picture of the fundamental processes of charge separation that follow an absorption event. We apply the formalism to two-level photovoltaic cells and our purpose is to pedagogically explain the main aspects of the model. The formalism is developed in the energy domain and provides detailed knowledge about existence or absence of localized states and their effects on electronic structure and photovoltaic yield.
\end{abstract}

\section{Introduction}

Nanostructured photovoltaic cells including organic solar cells, dye and quantum dot sensitized- solar cells have received great attention as promising photovoltaic technologies because of their prospective applications and interesting operation principle [1-12]. In nanostructured solar cells, following the photon absorption, electron-hole pair creation occurs in a confined zone [13-16] and hence, the Coulomb interaction between the charge carriers and their possible recombination plays an essential role. Fundamentally different from conventional silicon-based photovoltaic devices, in nanostructured solar cells the charge carriers separation and transport rely on a high density of nanoscale interfaces.

While understanding the performance of nanostructured solar cells has been a central effort of the scientific community for many years [17-21], theoretical approaches still are needed to facilitate the understanding of electron-hole interaction and recombination effects on the cell performance. In view of their nano-metric size, the semi-classical approaches are expected to fail and fully quantum treatments are needed.

We develop a new quantum formalism, which is based on quantum scattering theory and in particular on the Lippmann-Schwinger equation [22-24]. A non-equilibrium wave function, which describes the state of the working cell, is computed and allows determining all parameters of the cell and its performance. As an example, we consider two-level quantum model and show that the formalism

a e-mail: tahereh.nematiaram@neel.cnrs.fr provides a comprehensive framework to understand the effects of electron-hole interaction and recombination on the performance of photovoltaic systems.

\section{Theoretical method}

\subsection{Two-level photovoltaic systems}

The basic idea of our methodology is described through the example of two-level photovoltaic systems with the electron-hole interaction and non-radiative recombination. The two-level system characterized by the HOMO (highest occupied molecular orbital) and the LUMO (lowest unoccupied molecular orbital) can be in the permanent or in the transitory regime of illumination.

As an example, for the photovoltaic devices in the permanent regime of illumination, we consider the molecular photocells where the energy conversion process takes place in a single molecular donor-acceptor complex attached to electrodes. In the absence of photons, the whole system is in the ground state with filled valence bands and empty conduction bands. Under the flux of photons, absorption by the molecule creates electron and hole in LUMO and HOMO, respectively. Both charge carriers interact via the Coulomb potential and can be recombined in the molecule or can be transferred to their respective channels where they produce permanent photovoltaic current (see Fig. 1left).

The Bulk-Hetero Junction (BHJ) structure is an example of systems in the transitory regime. Indeed in the BHJ structure, following the absorption of a photon an 

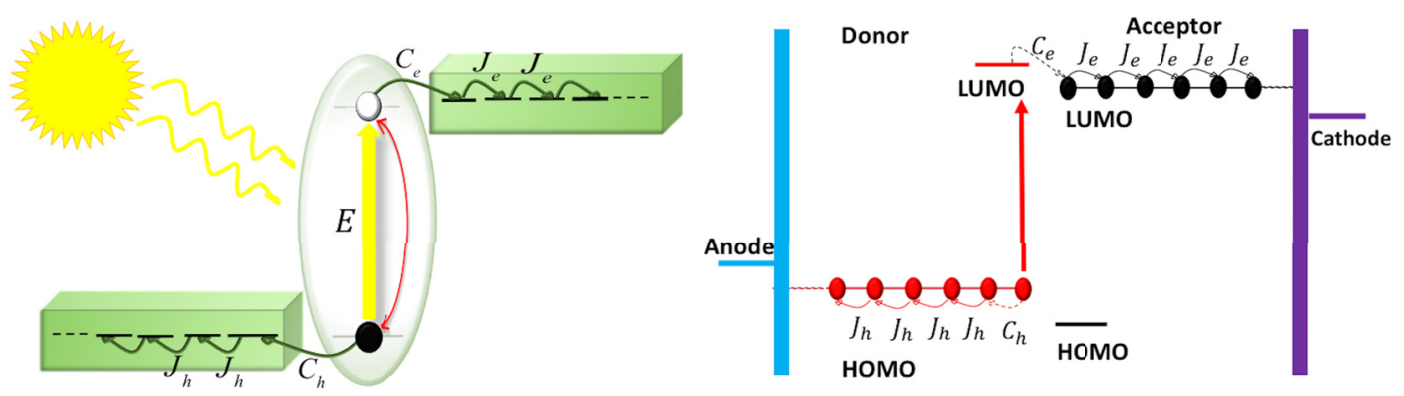

Fig. 1. The two-level model in the permanent (left panel) and transitory (right panel) regime of illumination. (Left panel) A molecular photocell with one HOMO and one LUMO orbitals attached to the electrodes. The red line represents the electronhole interaction and recombination inside the molecule and the hopping integrals of electron and hole are denoted by $C$ and $J . E$ is the energy of absorbed photon. (Right panel) Schematic representation of a donor-acceptor system. Charge evacuation leads are considered as semi-infinite chains. Here, $J_{e}$ and $J_{h}$ are the coupling energies between two adjacent sites in the electron and hole chains, respectively. Also, $C_{e}$ and $C_{h}$ represent the first coupling energies between the interface states and charge evacuation leads.

exciton is created in the donor side of the cell at one negative time, and then it diffuses up to the interface (the two-level system). This exciton arrives at the interface at time $t=0$ and our aim is to evaluate the total charge injected in each contact due to the exciton dissociation after a sufficiently large time (see Fig. 1-right).

\subsection{The electron-hole pair Hamiltonian}

In both systems of Figure 1, we have to determine the possible quantum states of the photo-generated electronhole pair. The Hilbert space of these electron-hole pairs can be mapped onto a square lattice (Fig. 2). For the PV devices in the permanent regime, $x(y)$ represents the position of the electron (hole), in the molecule or in the attached leads. Site $x=0(y=0)$ corresponds to the LUMO (HOMO) orbital in the molecule and $x>0(y>0)$ represents the electron (hole) position in its respective lead. Therefore, site $(x=0, y=0)$ is the electron-hole pair position on the molecule that is simply the initial state just after the photon absorption, i.e., the excited state.

Furthermore, for the PV devices in the transitory regime, $x(y)$ represents the position of the electron (hole), on the interface or in the attached leads. Site $x=0(y=0)$ corresponds to the LUMO (HOMO) orbital on the interface and $x>0(y>0)$ represents the electron (hole) position in its respective lead. Therefore, site $(x=0, y=0)$ is the electron-hole pair position on the D-A interface. The energy difference between LUMO and HOMO levels on the absorber molecule (permanent regime) or on the interface (transient regime) is equal to $\Delta$. The coupling matrix elements between the molecular or interface states and the possible evacuation leads is denoted by $C$. The hopping matrix element inside each evacuation lead is considered uniform and denoted by $J$. The onsite energies of the electron at site $(x)$ and the hole at site $(y)$ are assumed to be $\varepsilon_{e}(x)$ and $\varepsilon_{h}(y)$, respectively.

The effective Hamiltonian of the system is of the tightbinding type additionally including the electron-hole in-

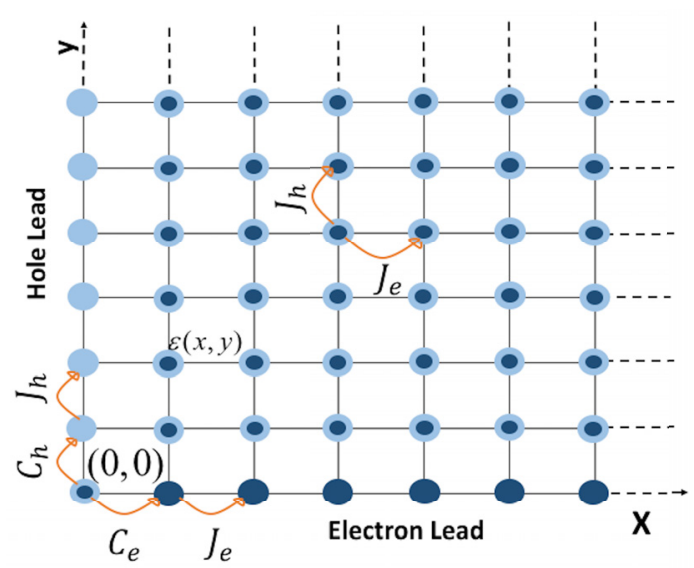

Fig. 2. The Hilbert space of the electron-hole pair by considering just one evacuation channel for each charge carrier, with one state at each point $(x, y)$ of the lattice. The coordinates $x$ and $y$ of a given state represent the position of electron and hole in their respective leads. $\varepsilon(x, y)$ is the onsite energy of each site of the square lattice and the hopping integrals $(C$ and $J$ ) are along the bonds of the square lattice.

teraction term

$$
H=\sum_{i} \varepsilon_{i}|i\rangle\left\langle i\left|+\sum_{i, j} J_{i j}\right| i\right\rangle\langle j|
$$

where the first term indicates the total onsite energy of each square lattice basis state, which is defined as a summation over the electron onsite energy, the hole onsite energy and the Coulomb interaction energy between them:

$$
\varepsilon(x, y)=\varepsilon_{e}(x)+\varepsilon_{h}(y)+I(x, y) .
$$

$I(x, y)$ represents the Coulomb-type interaction between the charge carriers and is modeled by:

$$
I(x, y)=\left\{\begin{array}{cl}
U & \text { if } x=0 \text { and } y=0 \\
\frac{V}{(x+y)} & \text { if } x \neq 0 \text { or } y \neq 0 .
\end{array}\right.
$$

Since $I(x, y)$ is an attractive Coulomb interaction, therefore $U$ and $V$ have negative values. 

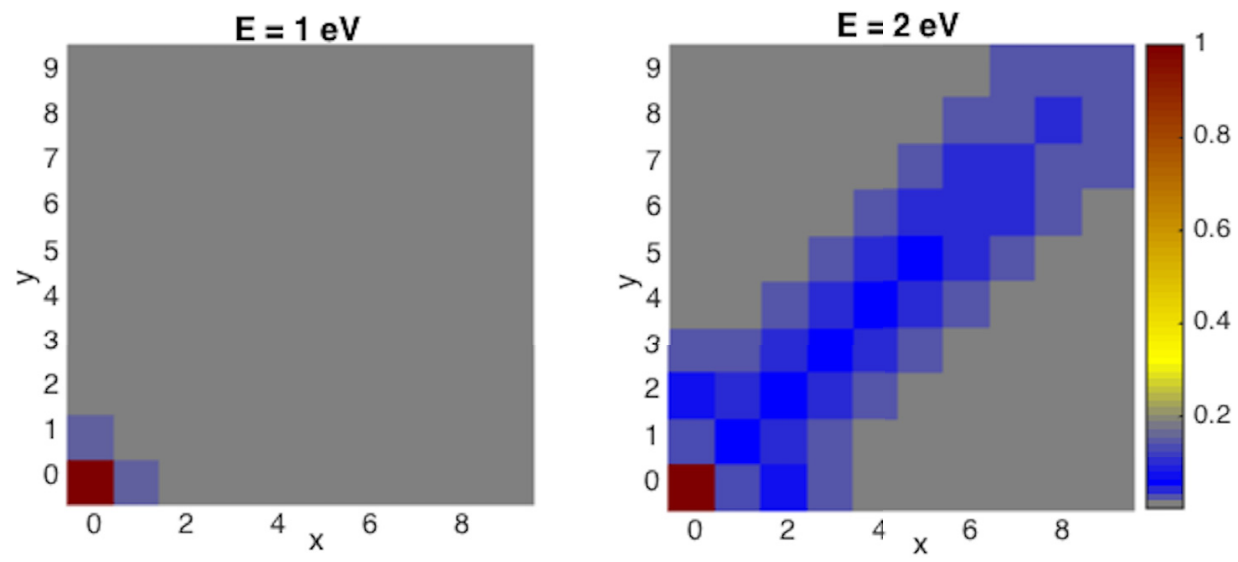

Fig. 3. Weight of the scattered wave function $\left(\left|\Psi_{p}(E)\right\rangle\right)$ on different sites $(x, y)$ of the square lattice normalized to its value on the initial site $\left(\left|\left\langle x, y \mid \Psi_{P}(E)\right\rangle\right|^{2} /\left|\left\langle 0,0 \mid \Psi_{P}(E)\right\rangle\right|^{2}\right)$ for various absorbed photon energies $(E)$. The energy continuum $(E C)$ is between 0.8 and $3.2 \mathrm{eV}$.

In equation (3), $U$ represents the strength of the local electron-hole interaction, i.e., when they are in the same place (either on the absorber molecule or on the D-A interface) and $V$ is the strength of the long-range electronhole interaction. In this formalism, the probability of photogenerated electron-hole pair local-recombination inside the absorber molecule or on the D-A interface is taken into account by adding an imaginary part $-i \Gamma_{R} / 2$ to the onsite energy of the site $(0,0)$, where $\Gamma_{R}$ is the recombination rate.

Finally, the second term in equation (1) represents the coupling energy between two adjacent basis states on the square lattice. As pointed above, the coupling parameters between molecular states or interface states (i.e., site $(0$, $0)$ ) and their first neighbors are taken different form the other coupling energies. The realistic values of hopping and interaction energies could be obtained from ab initio calculations or from experiments, however in this study they are taken as parameters.

\subsection{Non-equilibrium wave function of solar cell}

Let us first discuss the formalism for the case in the permanent regime. We consider a photovoltaic cell as a system submitted to an incident flux of photons and assume that the whole system (PV cell and electromagnetic field) is in a stationary state. By applying quantum scattering theory, an open quantum system method, in particular the Lippmann-Schwinger equation, the photovoltaic system is described by a wave function. The incoming state of the theory $\left|\Phi_{i n c}\right\rangle$ represents the photon field with the PV cell in its ground state. By the dipolar interaction between the photovoltaic system and the electromagnetic field this incident state $\left|\Phi_{i n c}\right\rangle$ is coupled to a state where one photon is absorbed and one electron-hole pair is created.

Based on the Lippmann-Schwinger equation and applying the Born approximation, the total wave function of the system is

$$
|\Psi(E)\rangle=\left|\Phi_{i n c}\right\rangle+\left|\Psi_{p}(E)\right\rangle .
$$

The second term in the right hand side of the above equation, $\left|\Psi_{p}(E)\right\rangle$, is called the scattered wave function which represents the charge carriers photo-generated by absorption of a photon with energy $E$ and plays an important role. $\left|\Psi_{p}(E)\right\rangle$ represents the whole system i.e., the cell with one electron hole pair plus the photon field with one less photon. Yet for the rest of this paper we focus on the current in the material system therefore we do not need to specify the part of $\left|\Psi_{p}(E)\right\rangle$ which represents the photon field. With this convention $\left|\Psi_{p}(E)\right\rangle$ is defined by the following equation:

$$
\left|\Psi_{p}(E)\right\rangle=\alpha(E) \frac{1}{E+i \varepsilon-H}|0,0\rangle
$$

where $\alpha^{2}(E)$ is the product of the square of the dipole matrix element of the molecular transition $d$ times the electromagnetic energy density $\rho(E)$ of photons with energy $E$, i.e., $\alpha^{2}(E)=d^{2} \rho(E) / 2 \varepsilon_{0} . \varepsilon_{0}$ is the vacuum permittivity, $\varepsilon$ is an infinitesimal positive energy and $|0,0\rangle$ is the excited state after the photon absorption. Furthermore, $H$ is the Hamiltonian of the electron-hole pair, which is defined by the tight-binding model on the mentioned square lattice.

To have an idea about the behavior of scattered wave function $\left|\Psi_{p}(E)\right\rangle$, Figure 3 represents the weight of $\left|\Psi_{p}(E)\right\rangle$ on different sites $(x, y)$ of the square lattice normalized to its value on the initial site $\left|\left\langle x, y \mid \Psi_{P}(E)\right\rangle\right|^{2} /\left|\left\langle 0,0 \mid \Psi_{P}(E)\right\rangle\right|^{2}$ by considering two different absorbed photon energies for a cell with energy continuum (EC) lying between 0.8 and $3.2 \mathrm{eV}$. It has to be noted that the energy continuum is the all allowed energies for the electron-hole pair. As can be seen, for the photon energies outside the continuum, the scattered wave function is nearly localized on the site $|0,0\rangle$ which is simply the absorber molecule. As the photon energy increases such that lies into the continuum, the scattered wave function extends on the various sites of the square lattice. 


\subsection{Fluxes}

In this formalism, the cell performance can be described through the definition of a series of fluxes and knowledge of scattered wave function $\left|\Psi_{p}(E)\right\rangle$ enables one to compute all the essential fluxes. The main three fluxes are: (1) the flux of absorbed photons $\Phi_{P h}(E)$ which is the number of absorbed photons per unit time; (2) the fluxes of electron-hole pairs that recombine in the molecule $\Phi_{R}(E)$; and (3) the flux of pairs that escape from the molecule and results in the photovoltaic current $\Phi_{C}(E)$. The flux conservation implies that

$$
\Phi_{p h}(E)=\Phi_{C}(E)+\Phi_{R}(E) .
$$

The flux of absorbed photons is related to the local DOS $n(E)$ through the Fremi's golden rule

$$
\Phi_{p h}(E)=\alpha^{2}(E) \frac{2 \pi}{\hbar} n(E),
$$

when the electron and the hole are not interacting $n(E)$ is nothing but the joint density of states between electrons and holes which is a key quantity for light-matter interaction. If the electron and the hole are interacting $n(E)$ is defined as the local density of states of electron-hole pair. This equation presents the direct relation between the photon absorption capability and the local density of states (LDOS) on site $|0,0\rangle$ of the square lattice. $n(E)$ can be expressed based on the diagonal matrix elements of the retarded Green's function

$$
n(E)=-\frac{1}{\pi} \operatorname{Im} G_{00}^{r}(E) .
$$

As will be discussed in the numerical method section, $G_{00}^{r}(E)$ can be expressed by:

$$
G_{00}^{r}(E)=\frac{1}{E+i \frac{\Gamma_{R}}{2}-\Sigma_{0}(E)}
$$

where $\Sigma_{0}$ and $\Gamma_{R}$ represents the self-energy of the system and recombination rate, respectively.

It has to be noted that the LDOS indicates all the possible energy states for the electron-hole pair and implicitly presents the capability of the photovoltaic cell to absorb the light. Additionally, the current and recombination fluxes can be expressed by

$$
\begin{aligned}
& \Phi_{C}(E)=\left|G_{00}^{r}(E)\right|^{2} \frac{\operatorname{Im} \Sigma_{0}}{\hbar} \\
& \Phi_{R}(E)=\left|G_{00}^{r}(E)\right|^{2} \frac{\Gamma_{R}}{\hbar} .
\end{aligned}
$$

Finally, the fluxes that allow describing the cell performance depend on recombination rate $\Gamma_{R}$ and self-energy $\Sigma_{0}$. The imaginary part of the self-energy $\Sigma_{0}$ plays a central role for the injection of current as shown by the above equation. The real part of the self-energy $\Sigma_{0}$ plays a central role to determine the position of the resonance in the density of states $n(E)$ and therefore in the frequency of photons that are most efficiently absorbed by the twolevel system. The self-energy can be determined from Hamiltonian of the square lattice (see numerical method).

\subsection{Expression of the quantum yield}

Let us first consider a photovoltaic cell in the permanent regime of illumination. At a given photon energy $E$, the yield $Y(E)$ is proportional to the ratio of photogenerated electrons or holes that arrive at the electrodes and the total number of absorbed photons at this given energy

$$
Y(E)=\frac{\Phi_{C}(E)}{\Phi_{p h}(E)}=\frac{-\operatorname{Im} \Sigma_{0}}{-\operatorname{Im} \Sigma_{0}+\Gamma_{R} / 2} .
$$

Through the assumption that $\alpha^{2}(E)=\alpha^{2}$ in the region where $n(E)$ (i.e., photon absorption) is important, the average yield or in other words, the charge separation yield $Y$, which is the proportion of the all electron-hole pairs, generated by different photons and giving rise to the photovoltaic current can be defined as

$$
Y=\frac{\int \Phi_{C}(E) d E}{\int \Phi_{p h}(E) d E}=\int n(E) Y(E) d E .
$$

Here, we draw the reader's attention to an interesting point: in the limit of small recombination rate, if photon energy $E$ lies into the energy continuum then there is a transformation into an electron-hole pair and therefore yield $Y(E)$ is one. Whereas, if $E$ corresponds to a bound electron-hole state, no current can be injected in the leads and finally the electron-hole pair recombine so that $Y(E)$ is zero. As a consequence, the average yield $Y$ given by equation (12) can be expressed by $Y=1-P$, with $P$ representing the total weight of localized states. This means that the efficiency of the cell depends critically on the existence or absence of the bound states as illustrated bellow. For the PV devices in the transitory regime, we consider the total charges injected in a bind (in units of the electron charge) $Q$

$$
Q=\int_{0}^{\infty} d t\langle\psi(t)|\hat{A}| \psi(t)\rangle
$$

where $\hat{A}$ is the current operator on that bind and

$$
|\psi(t)\rangle=U(t)|\psi\rangle ; \quad U(t)=e^{-i H t / \hbar},
$$

where $|\psi\rangle$ is the electron-hole pair wave function and $U(t)$ is the time evolution operator.

Through introducing the total electron number $Q_{e}$ or hole number $Q_{h}$ injected in the contacts one obtains

$$
Y=Q_{e}=Q_{h} .
$$

Interestingly, through the Fourier transform between the time and energy domain one obtains an expression for the charge separation yield in the transitory regime the same as equation (13).

\section{Numerical method}

\subsection{Recursion method}

In this section, we discuss the recursion method, which presents a central role in the computation of the scattered 

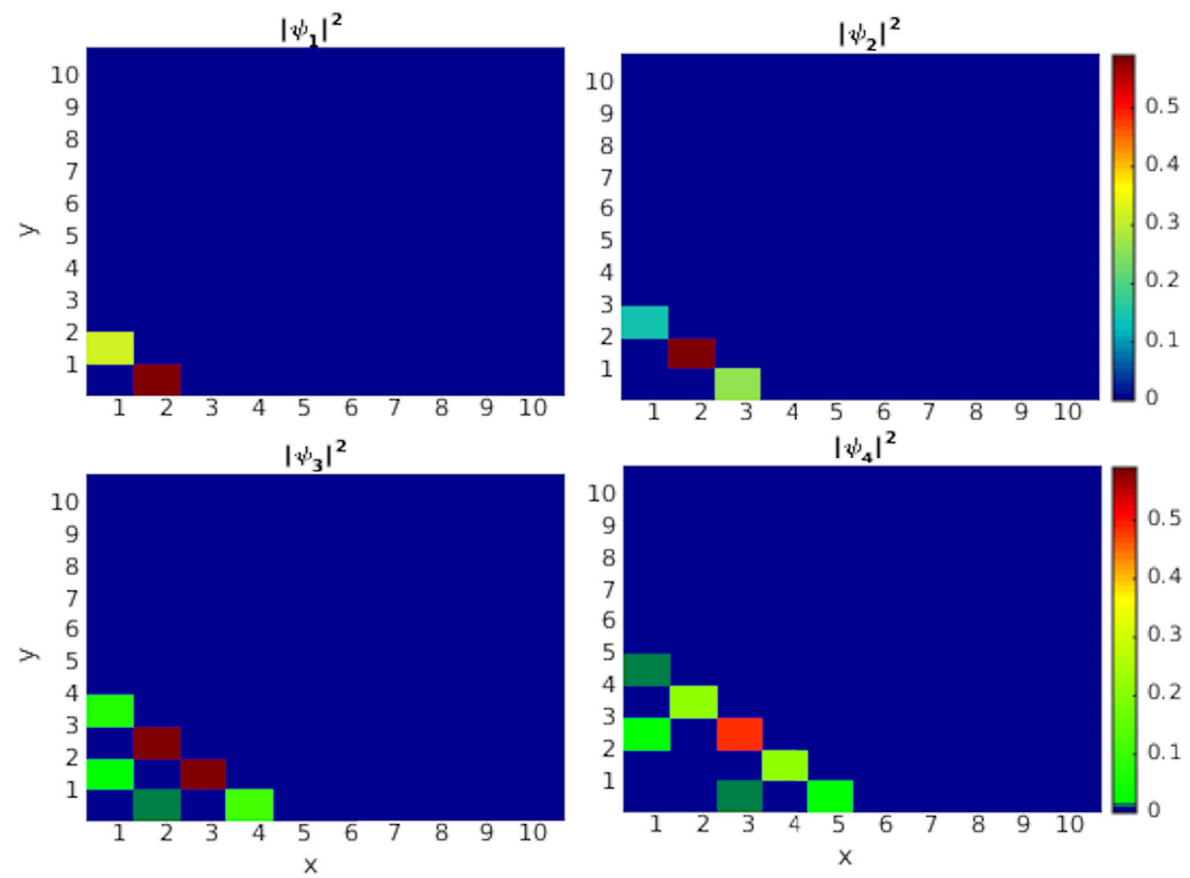

Fig. 4. Propagation of different orders of recursion wave-vectors. The coupling parameters are $J_{e}=J_{h}=0.3 \mathrm{eV}, C_{e}=0.2 \mathrm{eV}$ and $C_{h}=0.15 \mathrm{eV}$.

state and fluxes for a given Hamiltonian. An initial-state vector, on whose projection one going to compute a density of states, is selected. One can build a new basis set and a series of recursion coefficients starting from the mentioned initial state. In this new basis, the Hamiltonian becomes tri-diagonal and the recursion coefficients are the matrix elements of the Hamiltonian [25-28].

We consider a system described by a tight-binding Hamiltonian $H$. To a given normalized state $\left|\psi_{0}\right\rangle$ we can always associate a recursion basis which is constructed by a Schmidt orthogonalization procedure starting from the set of states $\left|\psi_{0}\right\rangle, H\left|\psi_{0}\right\rangle, H^{2}\left|\psi_{0}\right\rangle, \ldots, H^{n-1}\left|\psi_{0}\right\rangle, \ldots$ Let us consider $H\left|\psi_{0}\right\rangle$ and decompose it into two components: first component parallel to $\left|\psi_{0}\right\rangle$ and the second one orthogonal to $\left|\psi_{0}\right\rangle$. Therefore, we can write

$$
H\left|\psi_{0}\right\rangle=a_{0}\left|\psi_{0}\right\rangle+b_{1}\left|\psi_{1}\right\rangle .
$$

In the next step, we consider $H\left|\psi_{1}\right\rangle$ which can be decomposed into a component parallel to the space spanned by $\left|\psi_{0}\right\rangle,\left|\psi_{1}\right\rangle$ and a component orthogonal to this space. We obtain

$$
H\left|\psi_{1}\right\rangle=a_{1}\left|\psi_{1}\right\rangle+b_{1}\left|\psi_{0}\right\rangle+b_{2}\left|\psi_{2}\right\rangle
$$

The process can be repeated and leads to the construction of a set of states $\left|\psi_{n}\right\rangle$ which are orthonormal and satisfy the below equation

$$
H\left|\psi_{n}\right\rangle=a_{n}\left|\psi_{n}\right\rangle+b_{n-1}\left|\psi_{n-1}\right\rangle+b_{n}\left|\psi_{n+1}\right\rangle .
$$

The $a_{n}$ and $b_{n}$ parameters are called recursion coefficients and $\left|\psi_{n}\right\rangle$ are the recursion wave vectors. At any step $n$, the recursion coefficient $a_{n}$ is determined by $\left\langle\psi_{n}|H| \psi_{n}\right\rangle$ which is a real number. Then, the recursion coefficient $b_{n}$ and recursion wave vector $\left|\psi_{n+1}\right\rangle$ are obtained by the normalization condition for $\left|\psi_{n+1}\right\rangle$ and the choice of a real positive $b_{n}$. An important property of the states $\left|\psi_{n}\right\rangle$ is that they spread progressively from an initial state. In Figure 4, the propagation of recursion wave vectors on a square lattice is shown.

The significant achievement of the recursion method is that the Hamiltonian $H$ on the basis $\left(\left|\psi_{0}\right\rangle,\left|\psi_{1}\right\rangle, \ldots\right)$ can be written in a tri-diagonal form

$$
\hat{H}=\left(\begin{array}{cccccc}
a_{0} & b_{0} & 0 & \ldots & \\
b_{0} & a_{1} & b_{1} & & \ldots & \\
0 & b_{1} & a_{2} & b_{2} & \ldots & \\
\vdots & \ddots & \ddots & \ddots & \ddots \\
& & & & a_{n} & b_{n}
\end{array}\right) .
$$

Therefore, $G_{00}^{r}(E)$ can be written in the following form

$$
G_{00}^{r}(E)=\frac{1}{E+i \frac{\Gamma_{R}}{2}-a_{0}-\frac{b_{1}^{2}}{E-a_{1}-\frac{b_{2}^{2}}{E-a_{2}-} \cdot}}
$$

and in a shortened notation

$$
G_{00}^{r}(E)=\frac{1}{E+i \frac{\Gamma_{R}}{2}-\Sigma_{0}(E)}
$$

where, $\Sigma_{0}(E)$ is the self-energy as pointed previously.

Finally, let us recall that the recursion method allows the computation of the scattered wave function and of all 

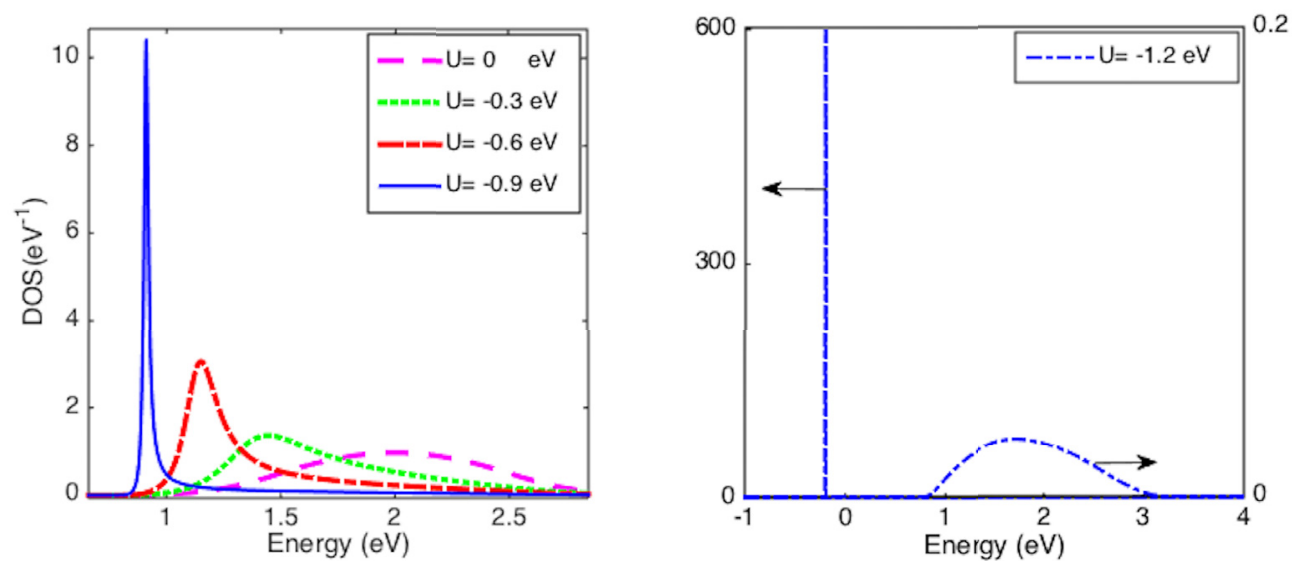

Fig. 5. LDOS as a function of the energy of the absorbed photon under the influence of short-range electron-hole interaction.

fluxes. Technically, one can compute $\left|\Psi_{p}(E)\right\rangle$ based on the product of recursion coefficients.

\section{Results and discussions}

\subsection{Study of occurrence of bound states under the influence of short-range electron-hole interaction}

In order to analyze the cell performance we consider the spectral information and in particular, the local DOS on site $(0,0)$. For the numerical calculation, parameters $\Delta=2 \mathrm{eV}, J_{e}=J_{h}=0.3 \mathrm{eV}, C_{e}=C_{h}=0.3 \mathrm{eV}$ have been used. With the chosen parameters the electron-hole pair energy continuum lies between 0.8 and $3.2 \mathrm{eV}$.

In Figure 5, the LDOS under the influence of shortrange Coulomb interaction conditions is plotted as a function of the absorbed photon energy. As can be seen for small values of $|U|$, there is a single peak which becomes narrower as $|U|$ increases and all weight of the DOS is in the continuum part (left panel). As $|U|$ increases, the DOS line-shape splits into two parts, a part inside the energy continuum and a sharp peak outside the energy continuum (right panel).

The narrow peak outside the continuum is called excitonic state, which suppresses the charge carrier injection to the energy continum and expedite the charge carriers recombination. The creation of excitonic state can be understood in full details through the discussion given in reference [21], which shows that there is a critical interaction energy to create localized states. Mathematically an excitonic peak appears at a given energy $E$ outside the energy continuum, if

$$
E-\Sigma_{0}(E)=0 .
$$

Indeed excitonic states are the poles of the retarded Gree's function. It is expected that in a bound-state the charge carriers will ultimately recombine since they cannot escape into the leads. The appearance of excitonic states decreases the number of charge carriers arrived at the electrodes and therefore lowers the photocell yield.
For the local interacting electron-hole pair, the dependence of the yield $Y$ on the electron-hole interaction $U$ for two different recombination rate $\left(\Gamma_{R}\right)$ and different set of coupling parameters $\left(C=C_{e}=C_{h}\right)$ is investigated in Figure 6. As can be seen, in all cases, for small values of interaction energy, the yield remains 1 for $\Gamma_{R}=0$. As $|U|$ increase, the yield decreases. This behaviour can be understood based on the information provided in Figure 5. For larger values of $|U|$, the charge carriers will stay on the molecule (or interface) to form a localized state because their energy does not lie in the energy continuum of the contacts. Furthermore, for large values of the coupling parameters $(C)$, charge carriers will transfer more efficiently to the evacuation channels and hence the cell remains efficient over a wider range of the recombination parameter $\Gamma_{R}$.

\subsection{Occurrence of bound states under the influence of long-range Coulomb interaction}

Now we turn to the case of the long-range electron-hole interaction and examine its impact on the spectral properties (see Fig. 7). We find that under the influence of longrange electron-hole interaction a series of excitonic peaks appears below the lower band of energy continuum. This is expected, as it is known that the long-range Coulomb attraction creates localized states. In this figure, all the excitonic peaks close to lower band edge cannot be resolved.

We see that, as the interaction strength increases, the weight of localized states increases as well. Therefore, under the influence of long-range interaction less charge carriers exit through the contacts because of localizedstate formation and hence it is expected that the yield decreases.

\subsection{Occurrence of bound states under the influence of lattice distortion and polaron formation}

The charge carrier mobility can be influenced by the electron-phonon vibration interaction and therefore in the 

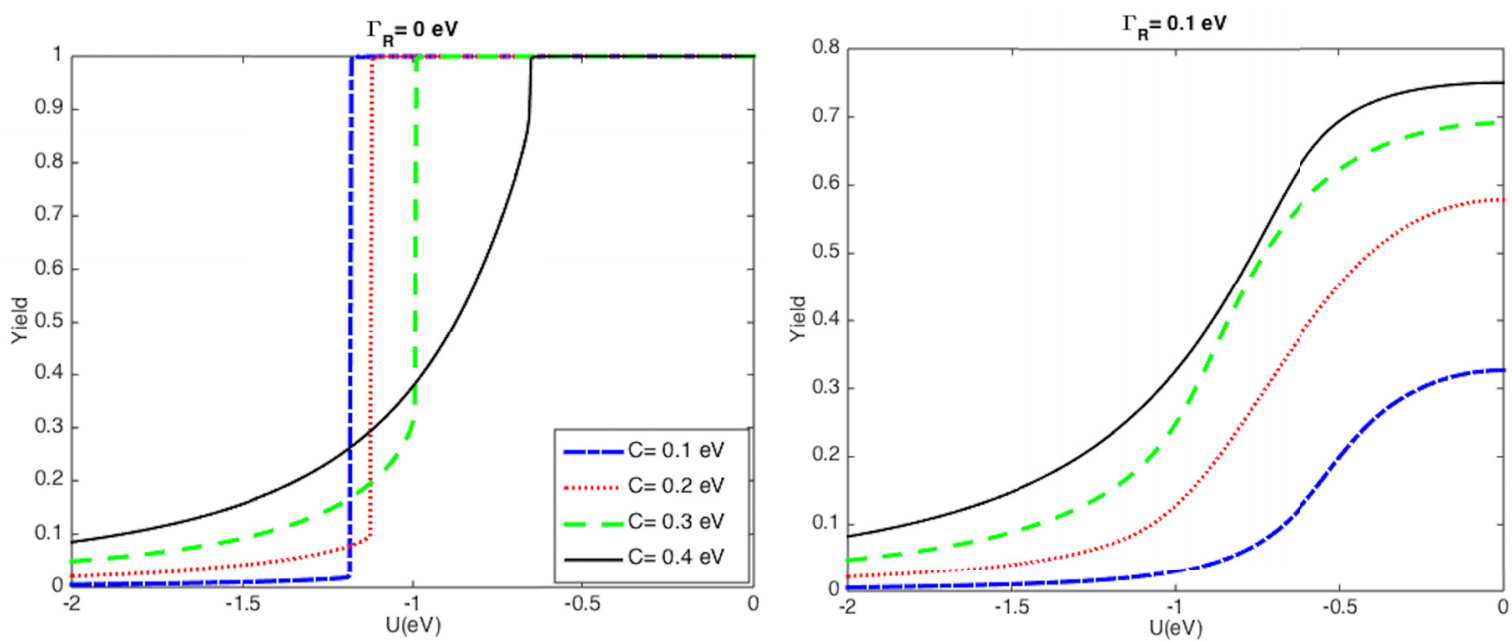

Fig. 6. Yield of a two-level photovoltaic cell as a function of interaction energy $(U)$ for different recombination rate $\left(\Gamma_{R}\right)$ and coupling parameter $(C)$.
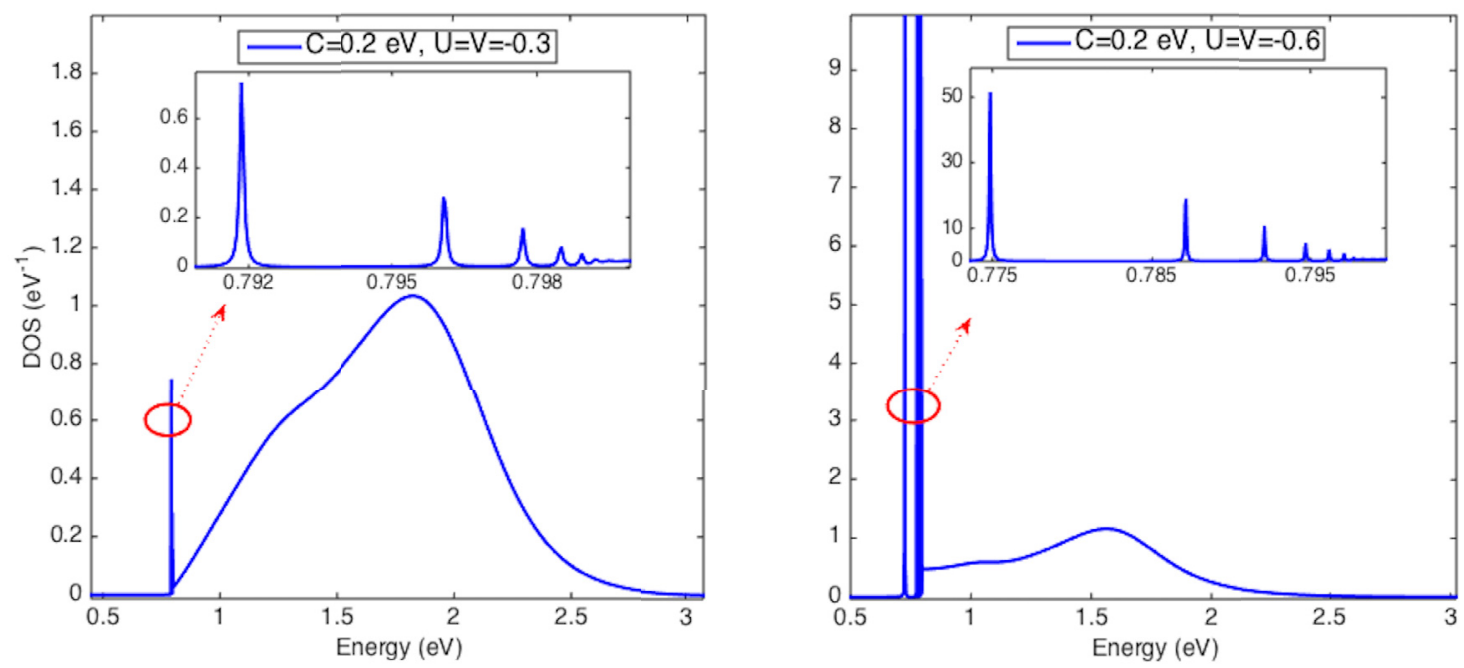

Fig. 7. LDOS as a function of the energy of the absorbed photon under the influence of long-range electron-hole interaction.

strong coupling regime, the charge carrier transport can be described by a polaron hopping from molecule to molecule where a polaron is a freely moving charge (electron or hole), surrounded by virtual cloud of phonons [29].

To have a clear idea, suppose in a D-A system, the exciton arrives at the interface at time $t=0$ coming from the donor side. After the exciton dissociation at the DA interface, the electron either will be recombined with the hole or will move through a set of acceptor sites. To investigate the effects of coupling to the phonon modes, we suppose that electron on each acceptor site can be coupled to one single phonon mode. The model considered here is exactly that described in [29].

Figure 8 represents the typical electronic structure of such a system far from interface (left panel) and on the interface (right panel). Based on the Holstein Hamiltonian model $\beta$ represents the strength of coupling to the phonon modes and $\varepsilon$ is the LUMO-LUMO offset [29]. As can be seen from the left panel, the electronic structure is com- posed of a series of bands called polaronic bands and an energy gap separates the different bands. Based on the right panel, for a given $\varepsilon$ the electronic structure may contain the energy states on the allowed polaronic bands as well localized states in the energy gap. The charge carriers lying in a polaronic band evacuate and arrive at the electrodes. On the other hand, the charge carriers localized in the bound state in the gap recombine quickly and cannot lead to photovoltaic current

\section{Conclusions}

To conclude, we have presented a non-equilibrium quantum formalism, which allows describing the fundamental phenomena related to charge separation or charge recombination after the absorption of a photon. This formalism should be useful in particular for nanostructured photovoltaic cells. 

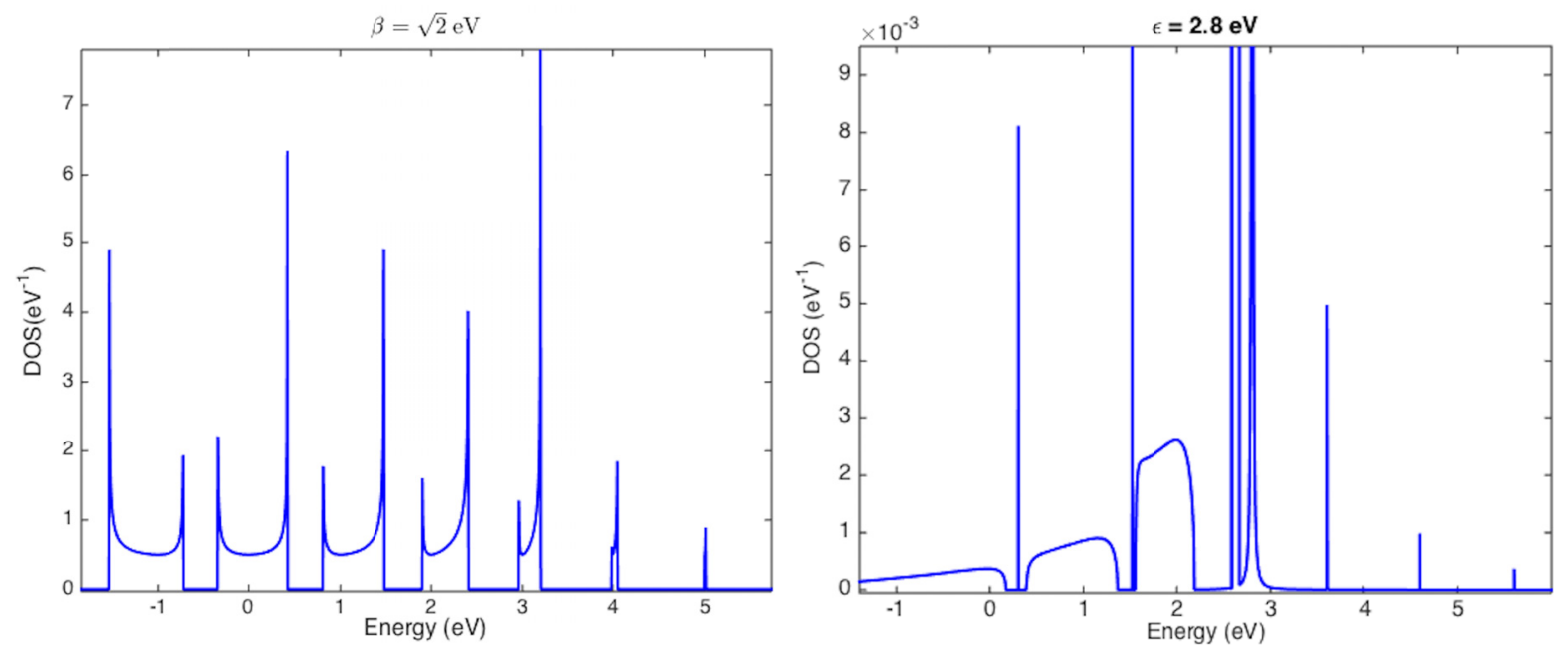

Fig. 8. Electronic structure as a function of the energy under the influence of coupling to the phonon modes. (Left panel) Far from the D-A interface. (Right panel) On the D-A interface. The hopping integral on the linear chain is $J=1 \mathrm{eV}[29]$.

In this paper, we considered the simplest case of a two-level quantum system This simple study illustrates the fact that localized excitonic or polaronic states can play a central role in the limitation of the quantum yield. This study represents that the formalism can be applied to include in particular the role of electron-hole interaction and the role of phonon on exciton propagation and charge separation.

The authors would like to thank Simone Fratini and KevinDavis Richler for fruitful discussions.

\section{References}

1. C.J. Brabec, Sol. Energ. Mat. Sol. Cells 83, 273 (2004)

2. C. Lungenschmied, G. Dennler, H. Neugebauer, S.N. Sariciftci, M. Glatthaar, T. Meyer, A. Meyer, Sol. Energ. Mat. Sol. Cells 91, 379 (2007)

3. M.T. Lloyd, J.E. Anthony, G.G. Malliaras, Mater. Today 10, $34(2007)$

4. M. Niggemann, B. Zimmermann, J. Haschke, M. Glatthaar, A. Gombert, Thin Solid Films 516, 7181 (2008)

5. J. Roncali, Acc. Chem. Res. 42, 1719 (2009)

6. F.C. Krebs, J. Fyenbo, M. Jørgensen, J. Mater. Chem. 20, 8994 (2010)

7. Y. Sun, G.C. Welch, W.L. Leong, C.J. Takacs, G.C. Bazan, A.J. Heeger, Nat. Mater. 11, 44 (2012)

8. Y. Li, Acc. Chem. Res. 45, 723 (2012)

9. A.J. Nozik, Physica E 14, 115 (2002)
10. A.J. Labelleet, Nano Lett. 15, 1101 (2015)

11. I. Chung, Nature 485, 486 (2012)

12. M. Liang, C. Jun, Chem. Soc. Rev. 42, 3453 (2013)

13. D. Cahen, G. Hodes, M. Gratzel, J.F. Guillemoles, I. Riess, J. Phys. Chem. B 104, 2053 (2000)

14. J.L. Brédas, J. E. Norton, J. Cornil, V. Coropceanu, Acc. Chem. Res. 42, 1691 (2009)

15. S. Ten Cate et al., J. Phys. Chem. Lett. 4, 1766 (2013)

16. F. Yang, S.R. Forrest, ACS Nano 2, 1022 (2008)

17. S. Ajisaka, B. Žunkovič, Y. Dubi, Sci. Rep. 5, 8312 (2015)

18. M. Einax, M. Dierl, A. Nitzan, J. Chem. Phys. 115, 21396 (2011)

19. M. Einax, A. Nitzan, J. Chem. Phys. 145, 014108 (2016)

20. M. Ernzerhof, M.A. Bélanger, D. Mayou, T. Nemati Aram, J. Chem. Phys. 144, 134102 (2016)

21. T. Nemati Aram, A. Asgari, D. Mayou, Europhys. Lett. 115, 18003 (2016)

22. J.J. Sakurai, Modern Quantum Mechanics (Addison Wesley, 1994)

23. T. Nemati Aram, P. Anghel-Vasilescu, A. Asgari, M. Ernzerhof, D. Mayou, J. Chem. Phys. 145, 124116 (2016)

24. T. Nemati Aram, M. Ernzerhof, A. Asgari, D. Mayou, J. Chem. Phys. 146, 034103 (2017)

25. F. Triozon, J. Vidal, R. Mosseri, D. Mayou, Phys. Rev. B 65, $220202(2002)$

26. P. Darancet, V. Olevano, D. Mayou, Phys. Rev. B 81, $155422(2010)$

27. D. Mayou, Europhys. Lett. 6, 549 (1988)

28. S. Roche, D. Mayou, Phys. Rev. B 60, 322 (1999)

29. S. Bera, N. Gheeraert, S. Fratini, S. Ciuchi, S. Florens, Phys. Rev. B 91, 041107 (2015) 